\title{
BRET-linked ATP assay with luciferase
}

\author{
Golnaz Borghei and Elizabeth A H Hall* \\ Received (in $X X X, X X X) X$ th $X X X X X X X X X 20 X X$, Accepted $X$ th $X X X X X X X X X 20 X X$ \\ DOI: $10.1039 / \mathrm{b000000x}$
}

${ }_{5}$ Taking advantage of BRET, a mutant firefly luciferase with higher $\mathrm{pH}$ - and thermo-stability than the wild-type could be coupled with the red-emitting fluorescent protein of mCherry in both a fused and unfused format. The BRET pair allows $>40 \%$ of the light emitted to be red shifted over $600 \mathrm{~nm}$ to the mCherry acceptor wavelength. Taking the expected quantum yield for mCherry $(0.22)$, a good fit to predicted light transfer is shown, with no other losses. Two measurements are considered for ATP 10 determination: a) a ratiometric technique for ATP measurement using both donor and acceptor emission intensities, making the calibration slope independent of protein concentration in a broad range. This measurement was limited by the BRET efficiency and the low quantum yield of the mCherry acceptor, but this detection limit might be improved with other fluorescent proteins with higher quantum yield. The fused BRET pair also resulted in a small increase in the BRET ratio. b) an ATP dependent shift in the 15 wavelength maximum using just the acceptor mCherry emission was also proposed for ATP determination. This did not require a high BRET efficieny and only uses emisson above $600 \mathrm{~nm}$ to obtain the acceptor emission maximum, but not its intensity; it is independent of protein concentration across a broad range. This offers a novel and robust method for determination of ATP between $10^{-11}-10^{-5} \mathrm{M}$ with an easy baseline calibration with ATP concentration $>10^{-4} \mathrm{M}$.

\section{${ }_{20}$ Introduction}

ATP, as the universal energy source for cellular function, is present in every type of biological cell and plays a critical role in energy exchange ${ }^{1}$. Therefore ATP measurement is crucial in analysis of cellular mechanisms, enzymatic processes and 25 biosynthesis $^{2}$. Furthermore, since all living organisms contain ATP, it finds widespread application in diagnostic assays of toxicity and contamination by microorganisms in varied fields such as the pharmaceutical industry, blood banks, food and water processing and environmental pollution ${ }^{3}$.

30 Among the simplest methods for ATP measurement are chromogenic and colorimetric techniques in which a result can often be read by eye ${ }^{1,4,5}$. However, probably the most promising and most studied approach to ATP measurement has been enzyme-linked. Since ATP is critical in numerous enzyme 35 pathways, there are many possibilities to exploit assays designed for a selective enzyme substrate, formatted so they are limited by the ATP concentration, rather than the primary substrate concentration. In this context, due to its high sensitivity and selectivity, the bioluminescent firefly luciferase (Fluc) -based 40 assay still remains the most widely used technique to measure $\mathrm{ATP}^{6}$. The firefly luciferase enzyme reaction has a fast response (milliseconds) and a broad range for ATP detection. Other adenosine-containing nucleotides such as AMP or adenosine diphosphate (ADP) do not react with the enzyme, so the reaction ${ }_{45}$ is highly specific to ATP. Theoretically sensitivity is extremely high, because there should be no background signal in the absence of ATP $^{2}$. Nevertheless, ATP measured down to the attomole level still requires low noise instrumentation ${ }^{7,8}$ to resolve the signal ${ }^{2}$ with any background signal due to extraneous so light eliminated 9 .

Another problem encountered in complex media and tissues which absorb a large proportion of the light below $600 \mathrm{~nm}$, is the short wavelength of emission for the luciferase-luciferin (Fluc$\left.\mathrm{LH}_{2}\right)$ system $(\sim 550 \mathrm{~nm})$. Attempts to shift the emission 55 wavelength through derivatisation of luciferin have been successful in terms of wavelength shift (eg the 6' amino derivatives have produced red-shifts up to to $625 \mathrm{~nm})^{10}$ but at the cost of light output, thus other ways to circumvent these limitations could be beneficial for some ATP assays.

60 Bioluminescence Resonance Energy transfer (BRET) is a natural phenomenon occurring in marine organisms such as Aequorea jellyfish and the sea pansy Renilla reniformis. It is a technique that has also been applied for noninvasive monitoring of BRET in live cells and whole-animal imaging ${ }^{11-13}$. Since the ${ }_{65}$ development of the first engineered BRET probe in $1998^{14}$, it has been widely used in analytical biosensing and imaging particularly via conjugation of luciferase enzymes as donors and fluorescent proteins as acceptors ${ }^{15-18}$. Despite red-emitting fluorescent proteins (RFPs) having the advantage of long 70 wavelength emission, use of red-emitting fluorescent proteins as acceptors in the general development of BRET systems is not as advanced and diverse as the use of GFP variants. The main reasons are that fewer monomeric RFP variants were available, as well as the lower quantum yield and brightness of these 
fluorescent proteins.

The most common application of BRET pairs introduced thus far has been in detection of protein-protein interactions

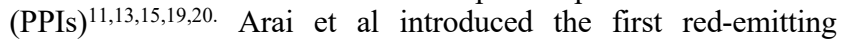
5 BRET system employed in a PPI, shortly after the discovery of RFPs. This used firefly luciferase as the donor and tetramer DsRFP as the acceptor ${ }^{21}$ (Quantum yield of DsRFP is 0.79). Among other successful luciferase-fluorescent protein fusion combinations that have been reported are Rluc-GFP ${ }^{22}$, Rluc10 mOrange $^{23}$, Fluc-mKate ${ }^{24}$, Fluc-mCherry ${ }^{25}$ and Rluc-EYFP ${ }^{13,26}$. Despite the reasonable performance of these BRET systems, most effort has been focused on their functionality as both an autofluorescent protein and a fluorescently tagged bioluminescent probe for in vivo imaging $12,17,22,23,26$. Noninvasive assesment PPIs 15 in cell cultures or tissues has always been of special interest due to their core role in understanding diseases and providing theraputic targets.

In analytical biosensing and imaging, bioluminescence and BRET have distinct advantages over fluorescence and Föster

${ }_{20}$ Resonance Energy Transfer (FRET). BRET does not require excitation of the donor by light, therefore shows significant lower background signal compared to FRET. Furthermore, the probability of undesirable direct excitation of the acceptor or photobleaching of the fluorophores, which are common 25 drawbacks in FRET, are insignificant in BRET. In tissue imaging applications, BRET is thus more applicable than FRET for photoresponsive cells (e.g. retina cells and most plant tissues) or autofluorescent tissues (containing molecules such as NADH, collagen or flavins). Direct excitation in FRET can damage the 30 tissue induced by a photogenerated chemical agent and adverse photochemical reactions. In addition, light sensitive pigments in photoresponsive tissues can react with specific wavelengths of light resulting in the activation of photosensitive biological processes.

35 Therefore, in a number of reports, BRET-based assays have been extended to study PPIs in living cells ${ }^{11,12,27,28 .}$. For example, Dragulescu-Andrasi et al have used BRET-based red-light emitting reporters to ratiometrically measured protein-protein interactions in deep-tissue small animal tumour models ${ }^{29}$. In 40 another work, "BRET3", a BRET-based probe composed of mOrange fluorescent protein $\left(\lambda_{\mathrm{Em}} 560 \mathrm{~nm}^{30}\right)$ and a mutant Renilla luciferase (RLuc8) employs red emission to observe biological signals from live single cells as well as from superficial and deeptissue structures ${ }^{31}$. Recently a fusion protein of an enhanced YFP 45 variant, Venus, and the RLuc8 was developed, which offers a BRET-based ratiometric $\mathrm{Ca}^{2+}$ indicator ${ }^{26}$. However, this luciferase enzyme does not use ATP as cofactor.

On the other hand, fusion proteins of firefly luciferase with fluorescent proteins (Fluc-FP) do offer a dual colour protein with 50 the potential for developing a useful ratiometric ATP measurement from the bioluminescence properties of luciferase and the fluorescence properties of fluorescent proteins. Such a dual signal measurement may be able to overcome issues of variability in signal intensity causing erroneous results. In another 55 work, Branchini et al designed a sequential BRET-FRET system employing a firefly luciferase, red-emitting mKate fluorescent protein and nIR fluorescent dyes ${ }^{24}$. In this instance the ratiometric luminescent probe was used to assay protease activities.

Here we examine a BRET-based technique for ATP 60 measurement. A mutant firefly luciferase (x5) with higher $\mathrm{pH}-$ and thermostability than the wild-type luciferase (Fluc) ${ }^{32}$ was selected, coupled with mCherry ${ }^{30}$ as a BRET-based probe for
ATP measurement. The combination of $x 5$ Fluc with mCherry shifts some of the luminescent emission wavelength to the red, so ${ }_{65}$ that together with the greater $\mathrm{pH}$ - and thermostability for the $\mathrm{x} 5$ firefly luciferase mutant, this could offer potential benefits either in the fusion protein format or the unfused protein, for BRETbased ATP measurement techniques.

\section{Materials and Methods}

${ }_{70}$ D-luciferin, ATP and EDTA, were purchased from SigmaAldrich UK. Taq DNA polymerase and KOD Hot Start DNA Polymerase used for polymerase chain reaction (PCR), were purchased from Novagen. QuikChange II XL Site-Directed Mutagenesis Kit was purchased from Agilent Technologies and 75 applied as per manufacturer's protocol. Enzymes used for cloning (restriction enzymes, calf intestinal alkaline phosphatase, Antarctic phosphatase, T4 DNA ligase) and Quick Ligation kit were purchased from New England Biolabs and were used according to the manufacturer's instructions. Plasmid pET16b 80 (Novagen) containing x5 luciferase gene was a gift from Dr Erica Law, University of Cambridge. Plasmid pRSET B, containing the mCherry sequence, was kindly provided by Dr Allison Denis from Prof Gang Bao's team at Georgia Tech University. Epicurian Coli ${ }^{\circledR}$ XL10-Gold ultracompetent cells were 85 purchased from Stratagene and transformation was carried out as per manufacturer's protocol.

\section{Construction of mCherry-Fluc fusion protein}

The pRSET B vector encoding x5-luc was used as a template for mCherry-Fluc fusion protein construction. The vector was 90 subjected to site directed mutagenesis aimed to add two restriction sites of NdeI and BamHI for later insertion of the Fluc gene, while the stop codon was placed at the end of the sequence. The linker between mCherry and Fluc in the fusion protein consisted of the original seven aminoacid tail of mCherry, 95 inserted by Shaner et $\mathrm{al}^{30}$ (plus one extra aminoacid of His), giving a linker sequence of: Gly, Met, Asp, Glu, Leu, Tyr, Lys, His. The resultant sequence was confirmed using the DNA Sequencing Facility, Department of Biochemistry, University of Cambridge.

\section{Expression and purification of His-tagged proteins}

Before expressing the protein, BL21(DE3) E. coli containing the desired plasmid were grown overnight in a starter culture of 15 $\mathrm{ml}$ Luria Broth (LB). The culture was then transferred into 200 $\mathrm{ml}$ fresh LB medium; grown to mid-log growth phase $($ OD600 $=$ 1050.4 to 0.6 ) and induced with isopropyl $\beta$-D-thiogalactoside (IPTG). For expression of mCherry and x5-luc $1 \mathrm{mM}$ IPTG were added at $25^{\circ} \mathrm{C}$ for $8 \mathrm{hrs}$. The fusion protein of mCherry-Fluc (CL) was expressed at $25^{\circ} \mathrm{C}$ with $0.1 \mathrm{mM}$ IPTG resulting in the pure fusion protein of CL. Purity of proteins was analysed using 110 SDS/PAGE (results not shown).

Induced cells were collected by centrifugation ( $\mathrm{RC}-5 \mathrm{C}$ centrifuge, Sorvall) at 9,500 rpm and $4^{\circ} \mathrm{C}$ for $10 \mathrm{~min}$ using the $\mathrm{F} 10-6 \mathrm{x} 500 \mathrm{y}$ rotor. The cell pellet was frozen and kept at $-80^{\circ} \mathrm{C}$ overnight. All processes of cell lysis and protein purification were carried out at $1154^{\circ} \mathrm{C}$. Cell pellets were resuspended with $5 \mathrm{ml} / \mathrm{g}$ wet cells in the lysis buffer (10 mM phosphate buffer, $\mathrm{pH} 8.0,2.7 \mathrm{mM} \mathrm{KCl}, 0.3$ $\mathrm{M} \mathrm{NaCl}, 10 \mathrm{mM}$ 2-mercaptoethanol, $20 \%$ (v/v) glycerol, 1× 
EDTA- free protease cocktail inhibitor with $2 \%(\mathrm{v} / \mathrm{v})$ Triton X100 and $20 \mathrm{mM}$ imidazole) following by addition of 124 units/g wet cells benzonase $\AA$ nuclease. Thawed resuspensions were mechanically pressed and put through three freeze - thaw cycles.

5 All proteins were purified with the same technique previously described by Law et $\mathrm{al}^{32}$ using Ni-NTA agarose beads followed by PD-10 desalting columns. Protein concentrations were estimated with the Bradford method ${ }^{33}$ using the Coomassie Blue protein assay reagent kit from Pierce as per manufacturer's 10 protocol, with BSA as the standard.
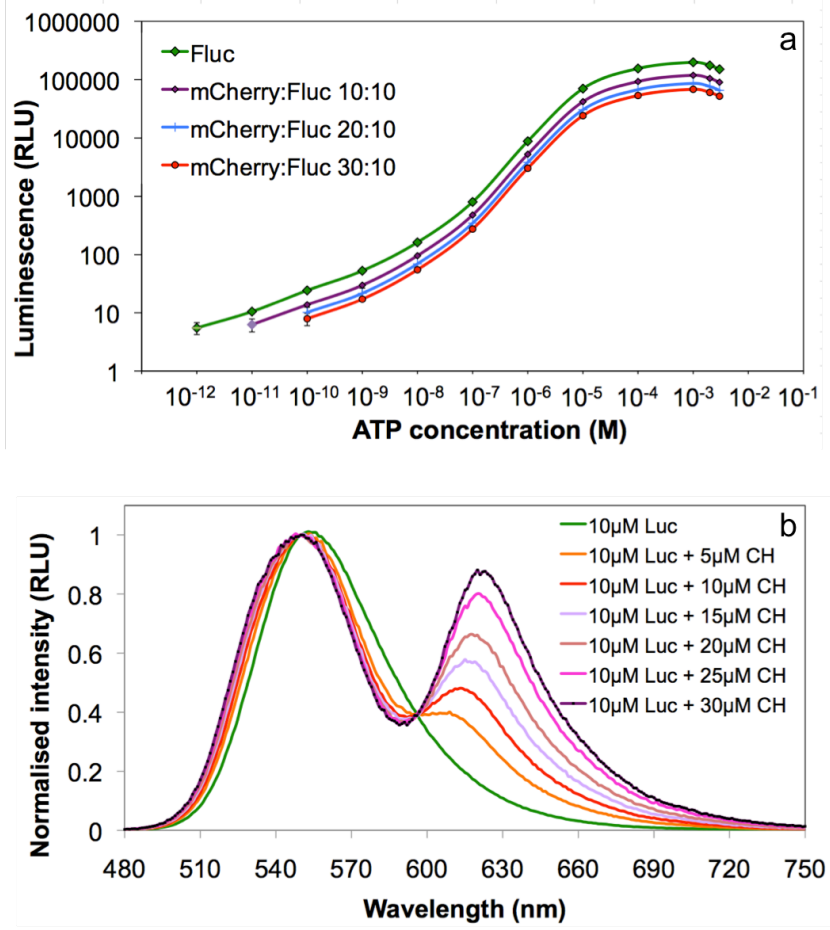

Fig.1 ATP measurement with BRET-based probes compared to $\mathrm{x} 5$ firefly luciferase. Luciferin concentration was $200 \mu \mathrm{M}$. Fluc concentration was fixed at $10 \mu \mathrm{M}$ in all probes whilst the mCherry concentration was varied. 15 (a) The concentrations used were: $10 \mu \mathrm{M} \times 5$ firefly luciferase, 10,20 and

$30 \mu \mathrm{M}$ mCherry. The y-axis shows the total emission output at $30 \pm 5$ seconds after ATP solution injection. Data were collected with a luminometer and correspond to relative total emission $>270 \mathrm{~nm}$. Bioluminescence measurements were carried out independently three 20 times. (b) Constant concentration of Fluc (Luc) at $10 \mu \mathrm{M}$, with increasing concentration of mCherry $(\mathrm{CH})$ from 5 to $30 \mu \mathrm{M}$. The spectra is normalised at the first peak around $550 \mathrm{~nm}$. The graph shows the increase in BRET ratio with the increase in the acceptor (mCherry) concentration.

\section{ATP measurement}

${ }_{25}$ Two probes were used to measure ATP based on BRET: (a) unfused mCherry and $\mathrm{x} 5$ Fluc in different concentration ratios of 5:10, 10:10, 20:10 and 30:10 mCherry:Fluc (Fluc concentration was fixed at $10 \mu \mathrm{M})$, (b) pure mCherry-Fluc (CL) fusion protein expressed at $25^{\circ} \mathrm{C}$. All probes were made in TEM buffer $(0.1 \mathrm{M}$

30 Tris/acetate, $\mathrm{pH} 7.8,10 \mathrm{mM} \mathrm{MgSO}_{4}$ and $2 \mathrm{mM}$ EDTA) containing $200 \mu \mathrm{M}$ D-luciferin $\left(\mathrm{D}-\mathrm{LH}_{2}\right)$. A range of fresh ATP solutions (picomolar to millimolar concentrations) was prepared in UHP water. The data were read with both luminometer (Labsystems Luminoskan Ascent luminometer with Ascent 35 Software) which collects all emission above $270 \mathrm{~nm}$ and fluorometer (Cary-Eclipse fluorescence spectrophotometer
(Varian)). The data were collected at $30 \pm 5$ seconds after the manual injection of ATP solution.

\section{BRET ratio calculation}

40 BRET ratio was measured with the fluorometer; the data were read at two peak-maxima wavelengths of typically $550 \pm 5 \mathrm{~nm}$ and $615 \pm 5 \mathrm{~nm}$. The BRET ratio calculations were obtained by dividing the emission intensity at the secondary (mCherry) peak maximum $(615 \pm 5 \mathrm{~nm})$ by emission intensity at the first (excited 45 state of $\mathrm{LH}_{2}$ oxidation product) peak maximum $(550 \pm 5 \mathrm{~nm})$.

\section{Results and Discussion}

\section{ATP linked emission with BRET pairs}

\section{Unfused mCherry and Fluc}

Figure 1a compares the luminescent intensity (all emission ${ }_{50}>270 \mathrm{~nm}$ ) for unfused BRET-probes of mCherry and Fluc in the presence of ATP and luciferin, for a constant concentration of Fluc; this shows a small decrease in overall light output with increase in mCherry concentration. This is connected with an emission profile that has shifted to longer wavelengths, consistent 55 with resonant energy transfer to mCherry (fig. 1b), so that this reduction could be linked with the quantum yield for the fluorescent protein being $<1$ (QY for mCherry $=0.22^{30}$ ). For example, in this comparison, Fluc has a limit of detection of $\sim 1$ pM ATP, but the lower total emission output with increase in 60 resonance energy transfer to the fluorescent protein raises the detection limit so that for $30: 10 \mu \mathrm{M}$ mCherry:Fluc the limit has increased to $0.1 \mathrm{nM}$.

To explore whether the losses can be attributed entirely to the QY of the flurorescent protein (FP), the mCherry emission output ${ }_{65}$ can be predicted by considering the reduction in the fluorescence at the FP emission, due to the quantum yield of the fluorescent protein $\left(\mathrm{QY}_{\mathrm{FP}}\right)$, from the intensity of Fluc emission in the presence of FP (B) and the absence of FP $\left(\mathrm{B}_{0}\right)$ at the same concentration, according to:

${ }^{70} I_{t o t}=B_{0}-\left(1-\frac{B}{B_{0}}\right) B_{0}+\left(1-\frac{B}{B_{0}}\right) B_{0} Q Y_{F P}$

which allows the intensity of the fluorescent protein emission to be estimated according to:

$$
I_{F P}=\left(1-\frac{B}{B_{0}}\right) B_{0} Q Y_{F P}
$$

Assuming a QY for mCherry of 0.22 , Figure 2 demonstrates a 75 remarkable fit between predicted and experimental data for the mCherry+Fluc combination (increasing mCherry Figure 2A).

This also applies when the overall protein concentration is changed for a given mCherry:Fluc ratio (Figure $2 \mathrm{~B}$ for $1: 1$ ratio), indicating that there are no 'dark quenching' reactions between 80 the fluorescent protein and Fluc, reducing the total light output. This infers that the total luminescence and the BRET ratio $\left(\mathrm{I}_{\mathrm{FP}} / \mathrm{I}_{\mathrm{Fluc}}\right)$ are predictable, knowing the concentration and quantum yield of the acceptor, once the Stern-Volmer $\left(\mathrm{K}_{\mathrm{SVBL}}\right)$ constant for the BRET pair is known (figure 3C):

${ }_{85}\left(1-\frac{B}{B_{0}}\right)=\frac{1}{K_{S V B L}}$ 

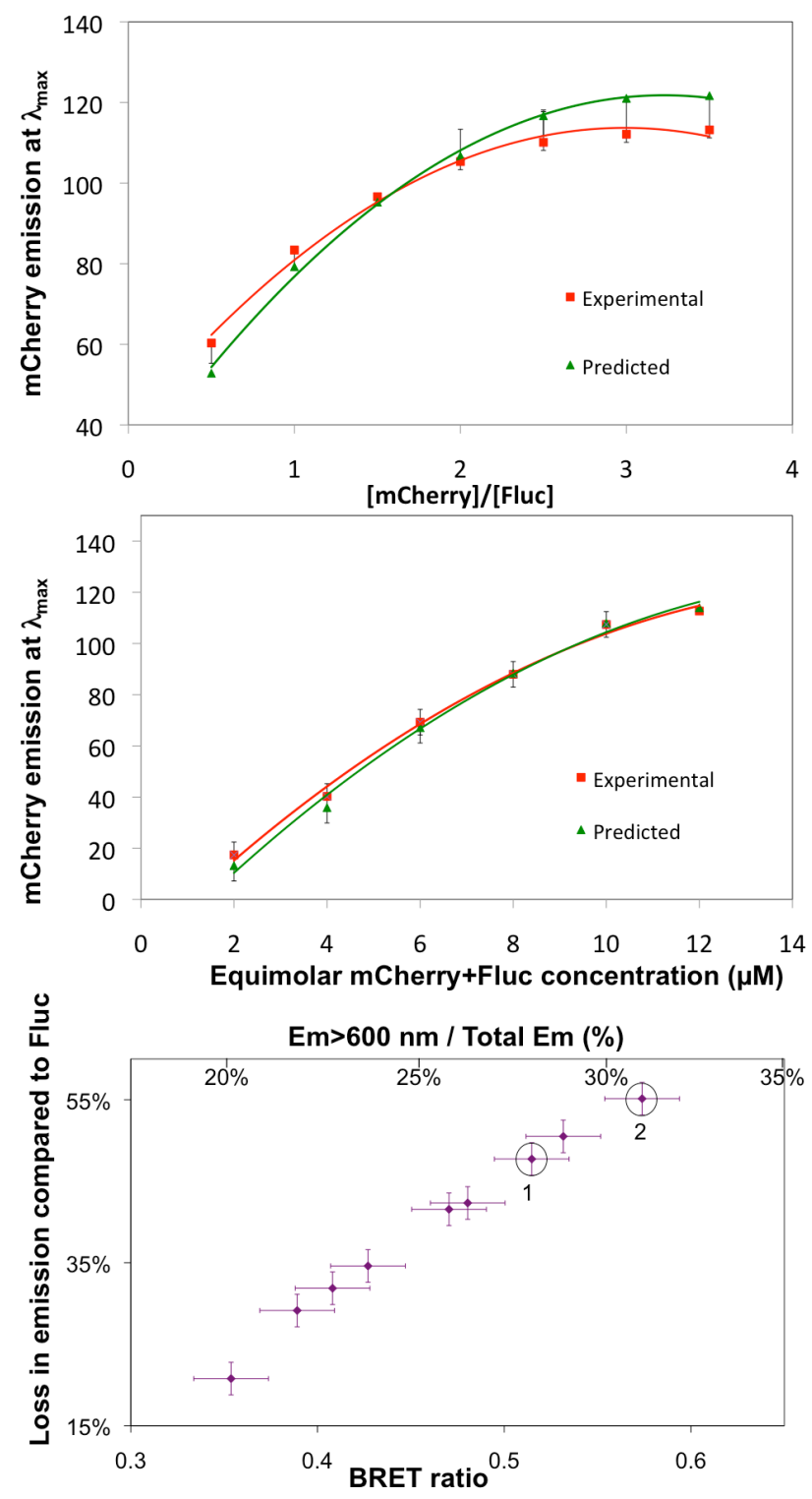

Fig.2 Predicted intensity at secondary peak (corresponding to mCherry emission) verses the experimental data. (a) constant concentration of Fluc at $10 \mu \mathrm{M}$, with increasing concentration of mCherry. (b) equimolar mCherry+Fluc from 2 to $12 \mu \mathrm{M}$, . This suggests that the fluorescence emission is accurately predictable Shows no hidden loss of energy during the BRET mechanism. (c) Relation between loss in total emission (compared to equimolar

10 Fluc), BRET ratio and percentage shift to above $600 \mathrm{~nm}$ in 1:1 combination of mCherry:Fluc (fusion protein and equimolar unfused proteins). By measuring the BRET ratio, the loss in total emission and the light shift can be predicted (see text). Black circles on the graph represent the maximum BRET ratio obtainable by: (1) equimolar

15 mCherry+Fluc (2) CL fusion protein.

\section{ATP measurement via the BRET ratio}

It follows from the discussion above that trying to increase resonance energy transfer is thwarted by losses due to the QY of the fluorescent protein. Thus, a lower [FP] yields a higher light

20 output and from the previous derivations, the ratio between the emission intensity due to donor and acceptor in the BRET pair is a function of ATP concentration, as can be seen visually in Figure 3a. Thus, while the decrease in ATP concentration results in a decrease in the overall emission from the donor (Fluc-substrate 25 complex) the BRET ratio increases (Fig. 3b) and the BRET ratio is a function of [acceptor]/[donor]. Depending on the acceptor concentration, lower detection is still limited by the QY for the acceptor. With this configuration, the BRET ratiometric measurement of ATP could be resolved down to 10-100 pM.

${ }_{30}$ Furthermore, for 10:10 - 10:25 mixtures, the slope of the BRET ratio calibration curve below $10^{-8} \mathrm{M}$ is independent of protein concentration, which makes it a robust method for ATP measurement.
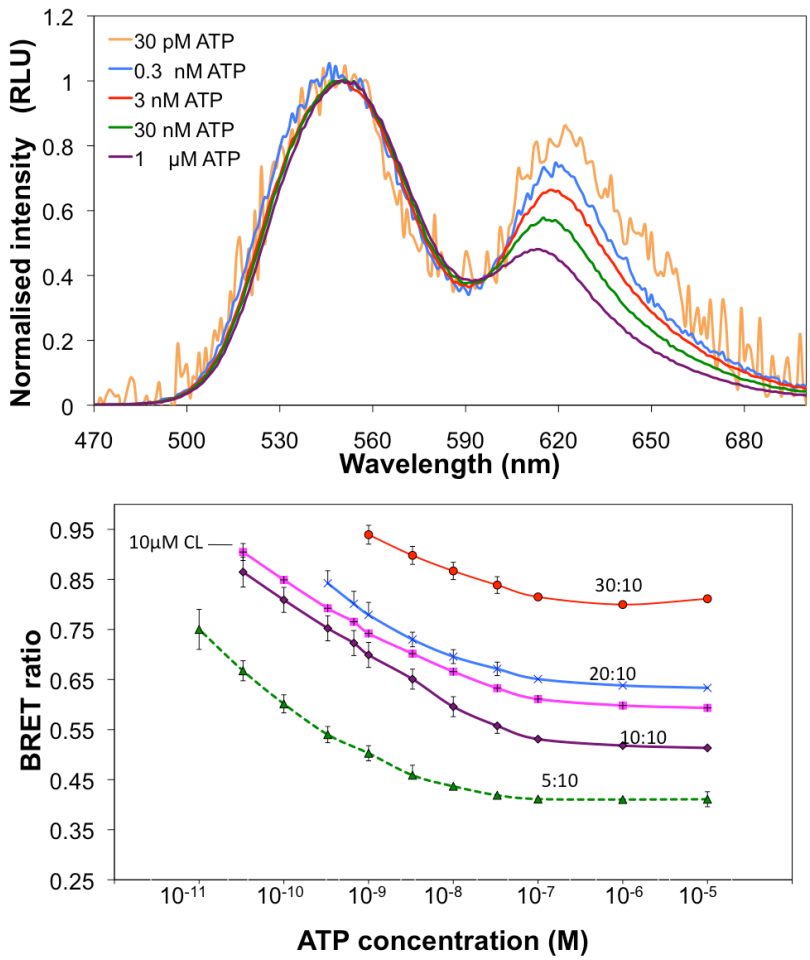

35 Fig. 3. (a) A BRET probe consisting 10:10 $\mu \mathrm{M}$ [mCherry]:[Fluc] unfused proteins. Normalised intensity at the BRET emission wavelengths with ATP concentration . (b) The change in BRET ratios as a function of ATP concentration for unfused BRET pairs with different ratios of

[mCherry]:[Fluc] with constant Fluc concentration at $10 \mu \mathrm{M}$ and mCherry 40 concentration from 5 to $30 \mu \mathrm{M}$. This is compared with the fused probe (CL) at $10 \mu \mathrm{M}$ mCherry-Fluc fusion protein. The trend is constant in different probes and the data is independent of sensitivity (PMT) of fluorometer. 

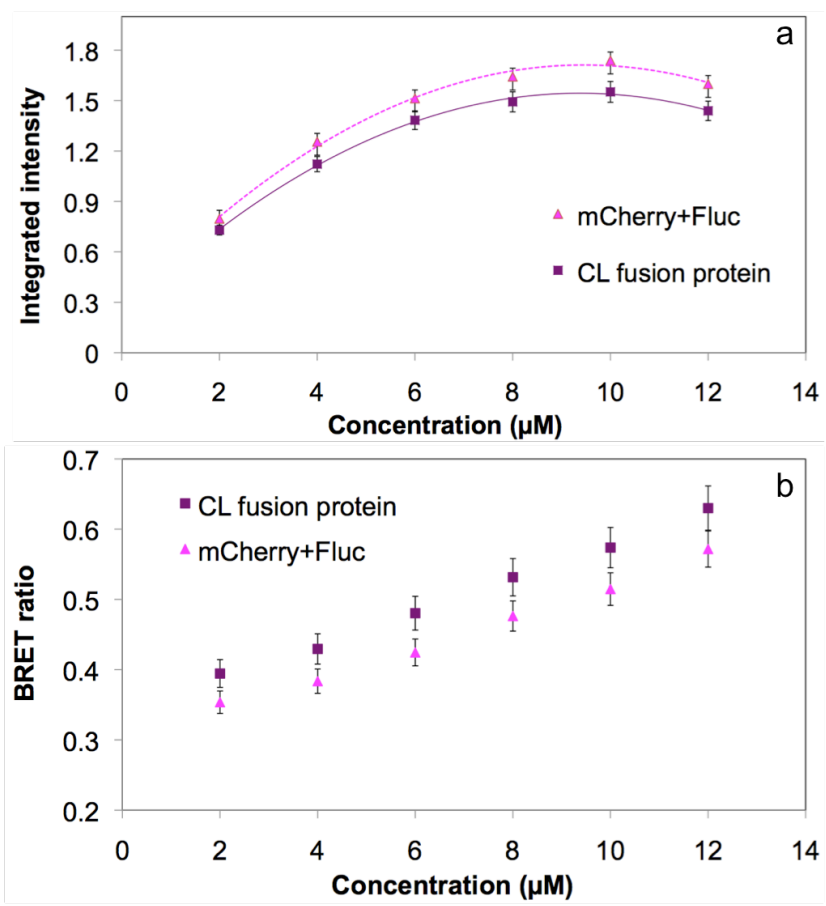

Fig.4 a) Relative integrated intensity and (b) BRET ratio of equimolar concentrations of CL fusion protein $(10 \mu \mathrm{M})$, in comparison with unfused equimolar of mCherry+Fluc $(10: 10 \mu \mathrm{M})$ are shown. $5 \%$ variations between experiments $(n=4)$. Data were collected with fluorometer. All proteins were expressed and purified under the same conditions as the fusion proteins $\left(25^{\circ} \mathrm{C}, 8 \mathrm{hrs}, 0.1 \mathrm{mM} \mathrm{IPTG}\right)$. Proteins were prepared in TEM buffer. The substrate consists of 200 $\mu \mathrm{M}$ D-luciferin, $1 \mathrm{mM} \mathrm{ATP}$ and $10 \mathrm{mM} \mathrm{MgSO}_{4}$. Data were at $30 \pm 5$

10 seconds after substrate injection.

\section{Fused mCherry-Fluc (CL)}

Based on this, it can be seen in Fig. 3b that a Fluc-mCherry fusion protein (CL) (with a forced intramolecular 1:1 ratio) follows a similar trend to the 10:10 mCherry:Fluc as would be 15 expected, but the ratios are distinct from the unfused counterpart. A comparsion between the total emission (Fig. 4a) and the BRET ratio (Fig. 4b) of a fusion protein of mCherry-Fluc (CL) and equimolar mCherry+Fluc confirms this and reveals the probable contribution of inter- and intra-BRET in the FP-Fluc pair. 20 Referring back to figure 2 (points 1 and 2) shows that the intensity of the emission above $600 \mathrm{~nm}$ can still be predicted according to equation 2, depending on the $\mathrm{QY}$ of the acceptor. However, as seen in Fig. 4a, a CL fusion protein with a controlled $1: 1$ ratio produces $\sim 10 \%$ lower luminescence emission compared 25 with the unfused proteins (Fig. 3b, 4b), but maintains the slope of the calibration curve as for the unfused proteins. To confirm this difference, a paired t-test was used (two-tailed, $\mathrm{df}=5$ ). $\mathrm{CL}$ fusion protein compared to unfused proteins shows p-value of $1.2 \times 10^{-5}$ with mean of the difference $=5.3 \times 10^{-2}$, suggesting a significant 30 difference between the BRET ratios with calculated p-values below 0.05 (95\% confidence interval). This does not show whether this is just due to a change in the geometric terms in the Förster equation as a result of the fusion, in particular $\mathrm{r}^{6}$ describing the inter-fluorophore distance or $\kappa$, describing the 35 dipole orientation, or some other change in Fluc kinetics. However, it does establish some vulnerability in an intensity based measurement for ATP.

\section{ATP measurement as a function of acceptor wavelength} maximum

40 It can be seen that at 10:10 $\mu \mathrm{M}$ Fluc:mCherry, $\sim 28 \%$ of the light has been shifted above $600 \mathrm{~nm}$ (Table 1), whereas a CL fusion protein achieves $\sim 32 \%$ and $10: 25 \mu \mathrm{M}$ Fluc:mCherry $\sim 40 \%$ above $600 \mathrm{~nm}$. This transfer of light above $600 \mathrm{~nm}$ is a significant advantage. However, for measurements in tissues, with 45 background autofluorescence below $600 \mathrm{~nm}$, the BRET ratio may still be compromised by needing the measurement of luciferase emission below $600 \mathrm{~nm}$ against this background for the ratiometic measurement. However, for ATP concentrations above $10^{-11} \mathrm{M}$, there is a well resolved wavelenth maximum for the BRET ${ }_{50}$ emission above $600 \mathrm{~nm}$ for all Fluc:mCherry or CL. This leads to another novel approach to a BRET measurement.

\begin{tabular}{|c|c|c|c|}
\hline $\begin{array}{l}\text { Concentration } \\
(\mu \mathrm{M})\end{array}$ & $\begin{array}{l}\text { Limit of ATP } \\
\text { detection (M) by }\end{array}$ & $\begin{array}{l}\text { Relative } \\
\text { ylight }\end{array}$ & $\begin{array}{l}\text { Photons above } \\
600 \mathrm{~nm} / \text { total }\end{array}$ \\
\hline & BRET ratio & output (\% & $(\%)$ \\
\hline 10 Fluc & $10^{-12}-10^{-13}$ & 100 & 13 \\
\hline $10: 5 \mu \mathrm{M}$ & $10^{-11}-10^{-12}$ & $75 \pm 5$ & $22 \pm 2$ \\
\hline Fluc:mCherry & & & \\
\hline $\begin{array}{l}\text { 10:10 } \mu \mathrm{M} \\
\text { Fluc:mCherry }\end{array}$ & $10^{-10}-10^{-11}$ & $55 \pm 5$ & $28 \pm 2$ \\
\hline $\begin{array}{l}\text { 10:20 } \mu \mathrm{M} \\
\text { Fluc:mCherry }\end{array}$ & $10^{-9}-10^{-10}$ & $45 \pm 5$ & $37 \pm 2$ \\
\hline $\begin{array}{l}\text { 10:30 } \mu \mathrm{M} \\
\text { Fluc:mCherry }\end{array}$ & $10^{-9}-10^{-10}$ & $34 \pm 5$ & $44 \pm 2$ \\
\hline $10 \mu \mathrm{M} \mathrm{CL}$ & $10^{-10}-10^{-11}$ & $50 \pm 5$ & $32 \pm 2$ \\
\hline
\end{tabular}

Table 1 Comparison of light emitted above $600 \mathrm{~nm}$ for different Fluc:mCherry combinations and CL fusion protein, normalised to totallight output by Fluc at the same concentration of $10 \mu \mathrm{M}$.

The idea that the fusion protein geometry highlights the energy transfer due to dipole or distance, leads to examination of the mechanism of the bioluminescence. Excitation to the oxyluciferin* state causes an increase in the dipole moment and 60 reverses its direction ${ }^{34,35}$ leading to disruption of bonds between oxyluciferin* and the enzyme binding site with partial dissociation of the enzyme-oxyluciferin* complex. Emission and deactivation can occur from either the associated or dissociated oxyluciferin ${ }^{* 36,37}$. The kinetics of this reorganisation causes ${ }_{65}$ conformational changes in the oxyluciferin* (including keto-enol tautomerism, scheme 1), which may result in a green or red shift in the fluorescent emission, imposed by interaction with the active site, which determines the final emission wavelength and peak broadening for a particular luciferase. 


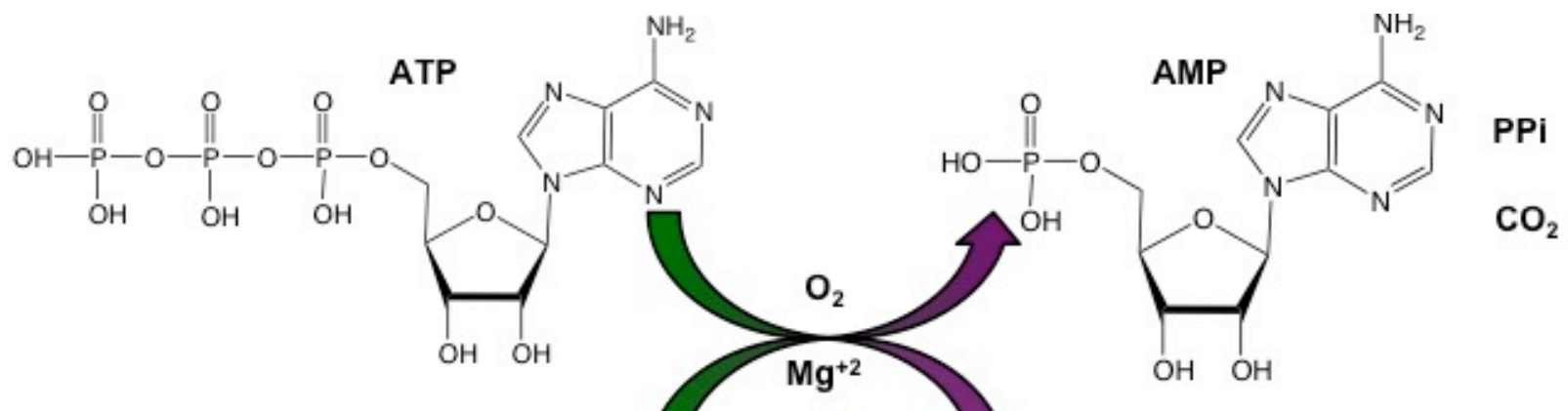

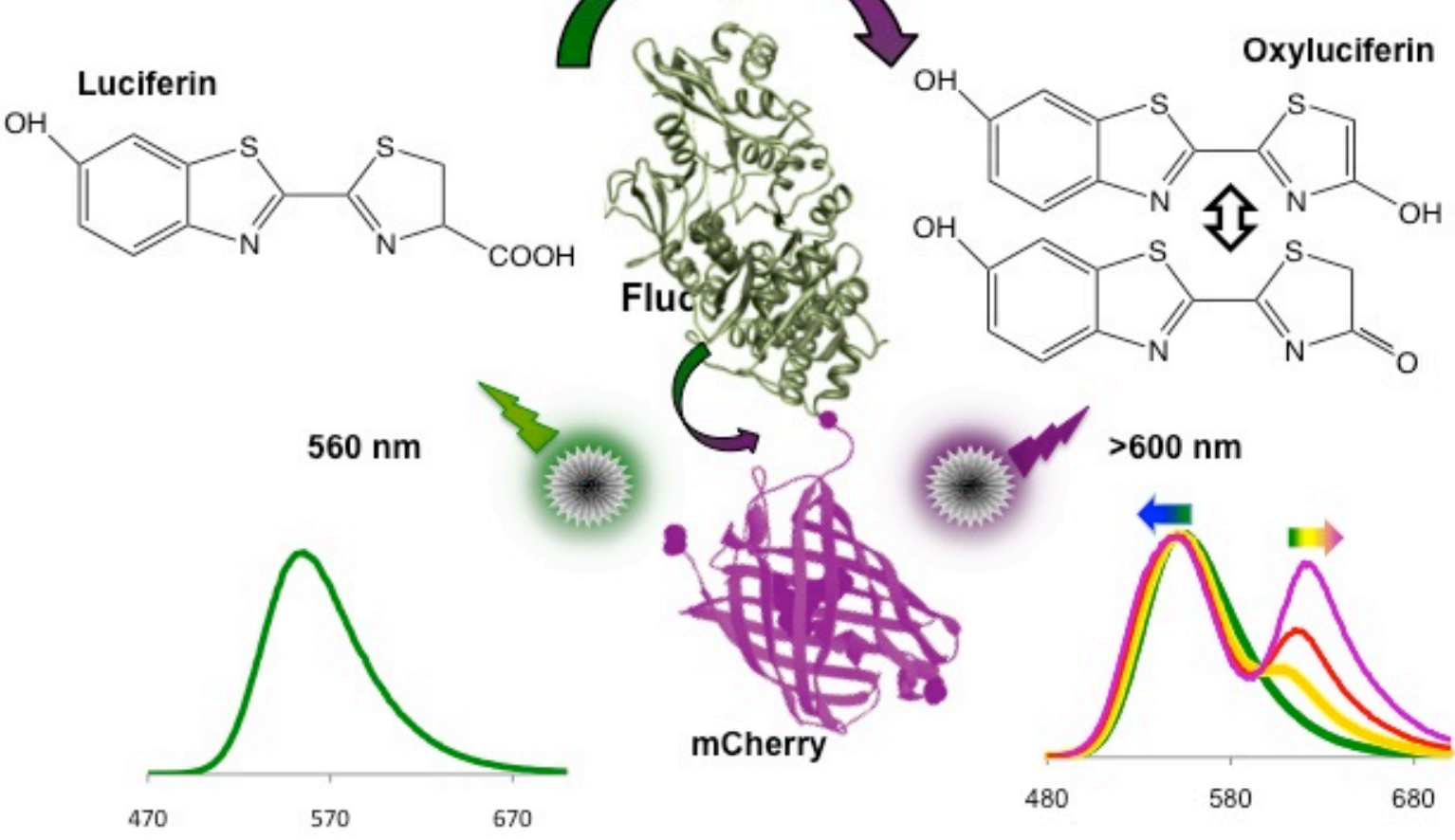

Scheme 1 BRET in Luciferase-Luciferin reaction: Luciferase acts as enzyme and donor, while mCherry is the acceptor. ATP measurement via the luciferase-luciferin signal (on the left) and the BRET pair signal due to the luciferase-luciferin donor and mCherry acceptor (on the right). Conformational changes in the oxyluciferin* (including keto-enol tautomerism), results in a green or red shift in the fluorescent emission

Rebartz et $\mathrm{al}^{38}$ has disentangled the keto-enol tautomerism for 10 oxyluciferin to identify the individual spectral contributors. This works suggests that the spectra range from the phenol-enol at low wavelengths through phenol-keto $<$ phenolate-enol $<$ phenolenolate $<$ phenolate-enolate to phenolate-keto at highest wavelength. These equilibria are dependent on protonation, 15 which in luciferase is served through the amino acid interactions in the active site. Since luciferase-bound luciferyl adenylate is one of the first intermediates in the mechanistic pathway, it might be postulated that at low ATP concentration, the conformational reorganization which results in changes in this equilibrium would 20 result in an ATP dependent product, and that the tautomer and its level of protonation could be revealed through measurement of the absorption spectra and possibly also the emission wavelength of the excited state oxyluciferin product.

The small blue shift in donor (Fluc) wavelength seen here, with 25 increase in ATP concentration is consistent with such a change in the keto-enol ratio due to the reorganisation of the oxyluciferin*, influenced by the concentration of ATP. However, the shift is small and not adequate to be correlated directly with [ATP].

On the other hand, the increase in ATP concentration is also 30 accompanied by a red-shift in the acceptor peak corresponding to mCherry fluorescence emission (Figure 5a). This is a much larger shift, which can be correlated to [ATP]. mCherry has an emission 
maximum of $611-613 \mathrm{~nm}$ when excited at $587 \mathrm{~nm}^{39}$, but as can be seen in figure $5 \mathrm{~b}$, the mCherry emission spectrum is dependent on excitation wavelength. At $10 \mu \mathrm{M}$ mCherry has a maximum emission $<614 \mathrm{~nm}$ when excited around 580nm (figure $5 \mathrm{~b}$ ), but 5 reached a maximum $621 \pm 1 \mathrm{~nm}$ when excited in UV Range $(<300$ $\mathrm{nm})$. So, we can correlate the shift in acceptor emission wavelength to a shift in excitation (donor) wavelength due to an ATP-dependent change in the oxyluciferin* reorganisation.
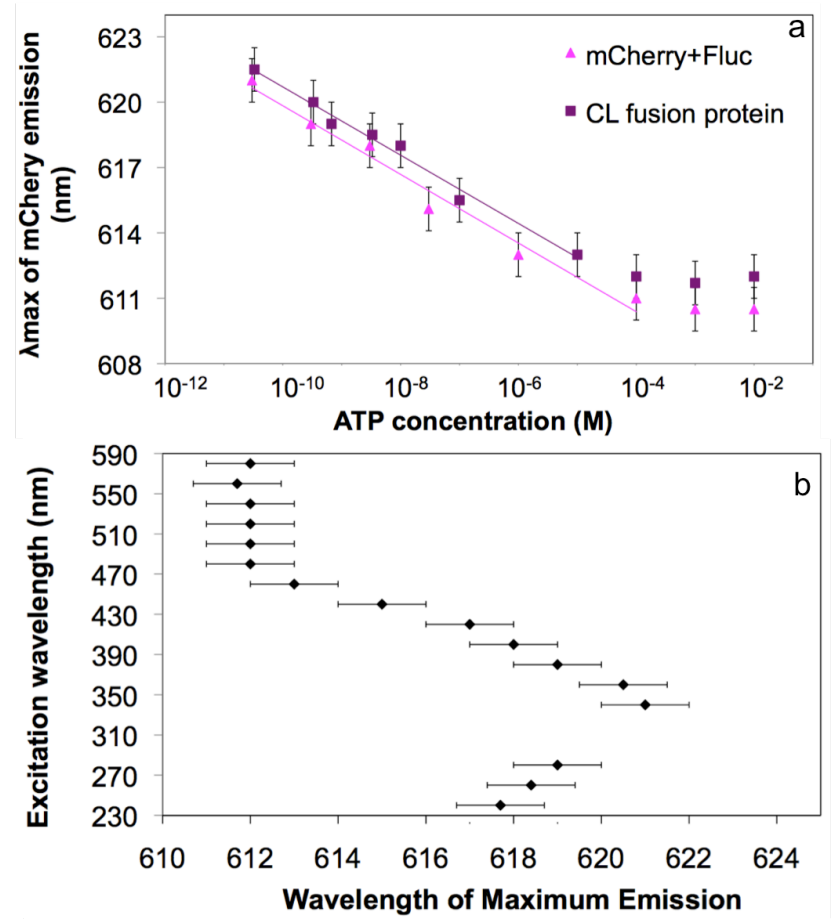

$10 \quad$ Fig.5 (a) The change in the wavelength of mCherry fluorescence maximum emission in a BRET pair of 10:10 $\mu \mathrm{M}$ [mCherry]:[Fluc] and

$\mathrm{CL}$ fusion protein. The graph shows a blue-shift in the maximum emission wavelength with an in increase in ATP concentration (donor emission) with constant acceptor concentration (Error: $\pm 1 \mathrm{~nm}$ ). (b)

15 mCherry $(10 \mu \mathrm{M})$ excited at different wavelengths. mCherry emission excited at UV range appears at longer wavelengths than excitation in visible range.

This leads to a robust ATP dependent calibration: like the BRET ratio measurement, the slope of the calibration curve for 20 mCherry $\lambda_{\max }$ VS ATP is independent of fused or unfused protein in the range $10^{-11}-10^{-5} \mathrm{M}$ ATP and has a 'baseline' calibration independent of concentration above $10^{-4} \mathrm{M}$ (figure 5a). This produces a calibration of:

$$
\begin{gathered}
\lambda_{o b s}-\lambda_{[A T P]>10^{-4} M}=x \\
{[\mathrm{ATP}]=10^{-(4+0.74 \mathrm{x})} \mathrm{M}}
\end{gathered}
$$

25

Importantly, this is the first ATP dependent measurement of BRET that can be performed exclusively above $600 \mathrm{~nm}$.

\section{Conclusion}

A BRET-based measurement of ATP suggests some potential 30 advantages over a conventional method employing pure Fluc. Emission above $600 \mathrm{~nm}$ becomes possible rather than the conventional pure firefly luciferase in which the emitted green light is largely absorbed by the tissue. By employing fused or unfused mCherry and Fluc, the BRET structure can transfer more 35 than $45 \%$ of the output light to wavelengths above $600 \mathrm{~nm}$ dependent on $[\mathrm{ATP}]$. However, on the down side, since the quantum yield for mCherry is only $0.22^{30}$, the BRET mechanism results in a significant overall intensity loss. Improved BRET efficiency with recently reported red-emitting fluorescent proteins 40 such as mKate $2^{40}$ could be a good alternative due to its high brightness ( 0.4 compared to 0.22 of mCherry), or eqFP650 can be used for a more red-shifted emission (Em max at $635 \mathrm{~nm})^{41}$, so that the BRET method does have a greater potential than achieved with this BRET pair.

45 Additionally, ratiometric measurement of the BRET donor and acceptor, as proposed here, provides a method that is independent of protein concentration within a broad range and resiliant to any protein loss with time. There is a higher energy transfer for the fusion protein of CL, probably associated with transfer distance 50 and dipole orientation and is attractive for in vivo measurement of ATP, where position of the members of a BRET pair may be difficult to manage otherwise.

Despite the advantages, a main drawback is the ongoing need to obtain a measurement at the lower Fluc wavelength as well as the 55 longer wavelength FP, so that a novel alternative approach that avoids intensity measurements, relating ATP concentration to the red shift in the mCherry emission wavelength, provides an interesting alternative for ATP measurement. We propose that the BRET pair allows fast energy transfer from an unstable high 60 energy green shifted conformer of the ATP-oxyluciferin*-enzyme complex, before its rearrangement. The result is that the BRET is dominated by this transfer at low [ATP] (below $\mathrm{K}_{\mathrm{M}}$ ), but at higher concentrations RET originates from the Fluc emission $>540 \mathrm{~nm}$. Since excitation wavelength dependent emission is also ${ }_{65}$ a feature seen in other FPs, there is potential for transfer to other FPs and better $\lambda_{\max }$ resolution at lower concentrations with the brighter FPs like mKate2.

\section{Notes and references}

Address, Institute of Biotechnology, Department of Chemical Engineering 70 and Biotechnology, University of Cambridge, Tennis Court Road, Cambridge, CB2 1QT.; Tel: 441223 334149; E-mail:

lisa.hall@biotech.cam.ac.uk

1 P. Mahato, A. Ghosh, S. K. Mishra, A. Shrivastav, S. Mishra, A. Das. 75 Inorganic Chemistry, 201150 (9), 4162-4170.

2 Z.Wang, P. G. Haydon, E. S. Yeung, Anal Chem 2000, 72, 20012007.

3 G. Borghei, E. A. H Hall ATP Measurement in Bio-Contamination. NATO Science for Peace and Security Series A: Chemistry and 80 Biology, ed. Nikolelis, D. P. Springer, 2012, 213-226.

4 A. Buryak, F. Zaubitzer, A. Pozdnoukhov, K. Severin, J Am Chem Soc 2008, 130, 11260-11261.

5 F. Sancenón, A. Descalzo, R. Martínez Máñez, M. Miranda, J. Soto, A Angew Chem Int Edit 2001, 40, 2640-2643

${ }_{85} 6$ P. Costa, N. Andrade, F. Passos, S. Brandão, C. Rodrigues, Brazilian Archives of Biology and Technology 2004, 47, 399-405.

7 B. R. Branchini, D. M. Ablamsky, A. L. Davis, T. L. Southworth, B. Butler, F. Fan, A. P. Jathoul, M. A. Pule, Anal Biochem 2010, 396, 290-297.

908 B. Rice, M. Cable, M. Nelson, J Biomed Opt 2001, 6, 432-440.

9 L. Ceresa, P Ball, Encyclopedia of rapid microbiological methods 2006, 2, 233-250.

10 G R Reddy, W C. Thompson, S. C. Miller. J. Amer. Chem.Soc. 2010, 132(39), 13586-13587

9511 A. De, S. S. Gambhir, FASEB J 2005, 19, 2017-2019.

12 A. De, A. M. Loening, S. S. Gambhir, Cancer Res 2007, 67, $7175-7183$. 
13 X. Xu, M. Soutto, Q. Xie, S. Servick, C. Subramanian, A. G Arnim, C. H. Von Johnson, Proceedings of the National Academy of Sciences 2007, 104, 10264.

$14 \mathrm{Y}$. Xu, D. Piston, C. A Johnson, Proceedings of the National Academy of Sciences 1999, 96, 151.

15 B. Breton, E. Sauvageau, J. Zhou, H. Bonin, C. Le Gouill, M. Bouvier, Biophysical Journal 2010, 99, 4037-4046.

16 A. A. Jensen, J. L. Hansen, S. P. Sheikh, H. Brauner-Osborne, Eur J Biochem 2002, 269, 5076-5087.

1017 D. Welsh, S. Kay, Curr Opin Biotech 2005, 16, 73-78.

18 Y.-P Kim, Z. Jin, E. Kim, S. Park, Y.-H. Oh, H.-S. Kim, Biochem Bioph Res Co 2009, 382, 530-534.

19 K. D. G. Pfleger, R. M. Seeber, K. A. Eidne, Nature Protocols 2006, 1, 337-345.

1520 K. D. G. Pfleger, R. D. Dromey, M. B. Dalrymple, E. M. L. Lim, W. T. Thomas, K. A. Eidne, Cell. Signal 2006, 18, 1664-1670.

21 R. Arai, H. Nakagawa, A. Kitayama, H. Ueda, T. Nagamune, J Biosci Bioeng 2002, 94, 362-364.

22 AA. Szalay, G.Wang, U.S. Patent US5976796, 1999.

2023 A. De, P. Ray, A. M. Loening, S. S. Gambhir, Faseb J 2009, 23, 2702-2709.

24 B. Branchini, J. Rosenberg, D. Ablamsky, Analytical Biochemistry 2011, 414, 239-245.

25 P. Iglesias, J. A. Costoya,. Biosens Bioelectron 2009, 24, 3126-3130.

2526 K. Saito, N. Hatsugai, Horikawa, K.; Kobayashi, K.; Matsu-ura, T.; Mikoshiba, K.; Nagai, T. PLoS ONE 2010 5, e9935.

27 Y. Xu, A. Kanauchi, A. Arnim, von, D. Piston, C. Johnson, Methods in enzymology 2003, 360, 289-301.

28 S. Gandor, S, Reisewitz, M. Venkatachalapathy, G. Arrabito, M.

30 Reibner, H. Schroder, K. Ruf, C. M. Niemeyer, P. I. H. Bastiaens, L. Dehmelt, Angew. Chem. Int. Ed. 2013, 52, 4790-4794.

29 A. Dragulescu-Andrasi, C. T. Chan, A. De, T. F. Massoud, S. S. Gambhir, PNAS 2011, 108, 12060-12065.

30 N. C. Shaner, R. E. Campbell, P. A. Steinbach, B. N. G. Giepmans, A. E. Palmer, R. Y. Tsien, Nat Biotechnol 2004, 22, 1567-1572.

31 A. De, P. Ray, A. M. Loening, \& S. S. Gambhir, Faseb J 2009, 23, 2702-2709.

32 G. H. E. Law, O. A. Gandelman, L. C. Tisi, C. R. Lowe, \& J. A. H. Murray, Biochem J 2006, 397, 305-312.

4033 M. Bradford, Anal Biochem 1976, 72, 248-254.

34 V. R. Viviani, T. L. Oehlmeyer, F. G. C. Arnoldi, \& M. R. BrochettoBraga, Photochem Photobiol 2007, 81, 843-848.

35 I. Navizet, Y.J. Liu, N. Ferre, H.Y. Xiao, W.H. Fang, and R. Lindh. J Am Chem Soc 2009, 132, 706-712.

4536 N.Ugarova, Photochem Photobiol Sci (RSC Publishing). 2008.

37 T. Sandalova, \& N.Ugarova, Biochemistry (Mosc) 1999, 926-927.

38 Rebarz, M., Kukovec, B., Maltsev, O. V., Ruckebusch, C., Hintermann, L., Naumov, P., Sliwa, M. Chemical Science 2013, 4, 3803-3809.

5039 D. Fink S. Wohrer M Pfeffer, T Tombe, CJ Ong, PH Sorensen, Genesis 2010, 48(12), 723-9.

40 D. Shcherbo, C. S. Murphy, G. V. Ermakova, E. A. Solovieva, T. V. Chepurnykh, A. S. Shcheglov, V. V. Verkhusha, V. Z. Pletnev, K. L. Hazelwood, P. M. Roche, S. Lukyanov, A. G. Zaraisky, M. W. Davidson, D.M. Chudakov, Biochem J 2009, 418, 567-574.

41 D. Shcherbo, I. I. Shemiakina, A. V. Ryabova, K. E. Luker, B. T. Schmidt, E. A. Souslova, T. V. Gorodnicheva, L. Strukova, K. M. Shidlovskiy, O. V. Britanova, A. G. Zaraisky, K. A. Lukyanov, V. B. Loschenov, G. D. Luker, D. M. Chudakov, Nat Methods 2010, 7, $827-\mathrm{U} 1520$ 